\title{
Long-term treatment of rheumatoid arthritis with adalimumab
}

This article was published in the following Dove Press journal:

Open Access Rheumatology: Research and Reviews

7 May 2013

Number of times this article has been viewed

\section{Giuseppe Murdaca \\ Francesca Spanò \\ Francesco Puppo}

Department of Internal Medicine, Clinical Immunology Unit, University of Genoa, Genoa, Italy
Correspondence: Giuseppe Murdaca Department of Internal Medicine, Clinical Immunology Unit, University of Genoa, Viale Benedetto XV,

16132 Genoa, Italy

Tel +39010 3537924

Fax +39010 5556950

Email giuseppe.murdaca@unige.it

\begin{abstract}
Rheumatoid arthritis (RA) is a chronic inflammatory disease that is associated with joint damage and progressive disability, an increased risk of morbidity related to comorbid conditions and substantial socioeconomic costs. Tumor necrosis factor-alpha (TNF- $\alpha$ ) is a proinflammatory cytokine known to have a central role in the initial host response to infection and in the pathogenesis of various immune-mediated diseases, such as RA, ankylosing spondylitis, psoriasis and/or psoriatic arthritis, Crohn's disease, and systemic lupus erythematosus. Five TNF- $\alpha$ inhibitors are available for the clinical use: infliximab; adalimumab; etanercept; golimumab; and certolizumab pegol. Infliximab is a chimeric human/murine IgG1 monoclonal antibody (mAb); adalimumab, and golimumab are human mAbs; certolizumab pegol is composed of the fragment antigen-binding anti-binding domain of a humanized anti-TNF- $\alpha \mathrm{mAb}$, combined with polyethylene glycol to increase its half-life in the body; etanercept is a fusion protein that acts as a "decoy receptor" for TNF- $\alpha$. In this paper, we will briefly review the current data on efficacy and safety of adalimumab in patients with RA, its potential beneficial effects upon comorbid conditions, such as endothelial dysfunction and accelerated atherosclerosis in RA, and the immunogenicity.
\end{abstract}

Keywords: adalimumab, efficacy, safety, rheumatoid arthritis, VEGF, immunogenicity, infections

\section{Introduction}

Rheumatoid arthritis (RA) is a chronic inflammatory immune-mediated disease that is burdened by progressive joint damage and disability, increased risk of comorbidity and socioeconomic costs. ${ }^{1-3}$ The ongoing progresses in the knowledge of the pathogenic mechanisms of various immune-mediated diseases, such as RA, ankylosing spondylitis (AS), psoriasis (Ps) and/or psoriatic arthritis (PsA), Crohn's disease (CD), systemic lupus erythematosus (SLE), and the availability of innovative biotechnological approaches, have led to the development of new drugs that add to conventional treatments. In particular, efforts have been made to design biologic drugs that are able to counteract the activity of different molecules (ie, tumor necrosis factor- $\alpha[$ TNF- $\alpha]$, interleukin 1 (IL-1), CD20, CD22, and CD11a). TNF- $\alpha$ is a proinflammatory cytokine known to have a central role in the initial host response to infection and in the pathogenesis of the above-mentioned diseases. ${ }^{4} \mathrm{TNF}-\alpha$ inhibitors have demonstrated efficacy in large, randomized controlled clinical trials either as monotherapy or in combination with other anti-inflammatory or disease modifying antirheumatic drugs (DMARDs). ${ }^{5-10}$ Five TNF- $\alpha$ inhibitors are available for the clinical use: infliximab, adalimumab, etanercept, golimumab, and certolizumab pegol. All these agents block the biologic effects of TNF- $\alpha$, although there are some differences in 
their structure, pharmacokinetics, and mechanisms of action. The efficacy and safety profile of the TNF- $\alpha$ inhibitors can be considered, in general, as a class effect. Nevertheless, some differences may exist among the five agents. Infliximab is a chimeric human/murine IgG1 monoclonal antibody (mAb), adalimumab, and golimumab are human mAbs, certolizumab pegol is composed of the fragment antigenbinding (Fab) domain of a humanized anti-TNF- $\alpha$ mAb combined with polyethylene glycol to increase its half-life in the body, etanercept is a fusion protein that acts as a "decoy receptor" for TNF- $\alpha .^{5-12}$ In particular, adalimumab is a fully recombinant human IgG1 anti-TNF- $\alpha$-specific $\mathrm{mAb}$ which is approved for the treatment of Ps, PsA, RA, AS, and CD. ${ }^{5-12}$ However, the efficacy and safety of adalimumab administered as monotherapy or in combination with methotrexate (MTX) for the treatment of RA has been well-established in clinical trials. ${ }^{13-19}$ In this paper, we will briefly review the current data upon efficacy and safety of adalimumab in patients with RA, its potential beneficial effects upon comorbid conditions, such as endothelial dysfunction and accelerated atherosclerosis in RA, and the immunogenicity.

\section{Efficacy and safety of adalimumab: how to optimize the treatment of RA}

Adalimumab is a fully recombinant human immunoglobulin G1 (IgG1) anti-TNF- $\alpha$-specific mAb that is capable of complement fixation and fragment crystallizable-(Fc) receptor binding. The plasma half-lives of antibodies appear to be largely related to the binding of their Fc regions to the neonatal Fc receptors (FcRn) on endothelial cells. The long plasma half-life of adalimumab suggests that it binds to FcRn like natural IgG1 molecules. Adalimumab is usually administered subcutaneously (40 mg every other week)..$^{5-12}$ Adalimumab was approved by the US Food and Drug Administration (FDA) in 2002 and was granted approval from the European Medicines Agency (EMA) in September 2003 for the treatment of RA.

Adalimumab was subsequently approved by the FDA for the following indications: PsA (in 2005), AS (in 2006), and CD (in 2007), as well as for juvenile-idiopathic RA and chronic plaque Ps, (both in 2008). Adalimumab is also approved for the treatment of these diseases by the EMA. ${ }^{5-12}$ Patients with RA must meet the following criteria before TNF- $\alpha$ inhibitors can be administered:

1. failure to respond to an adequate trial of at least two DMARDs, including MTX at an optimal dose (at least
$15 \mathrm{mg} /$ week and maximum dose $25 \mathrm{mg} /$ week) for a minimum of 3 months, or intolerance for MTX;

2. clinical evidence of active disease (multiple actively inflamed joints and/or Disease Activity Score uses 28 joint counts [DAS28] >3.2);

3. persistently elevated inflammatory markers, such as erythrocyte sedimentation rate (ESR) and C-reactive protein (CRP). ${ }^{8,20}$

Notably, DAS28 has been widely used to monitor the disease activity of patients with RA. The DAS28 cut-off points of 2.6, 3.2, and 5.1 have been proposed to be indicative of remission, low-disease activity and high-disease activity, respectively. ${ }^{8,21}$ In particular, treatment is judged effective in case of improvement of the DAS $28 \geq 1.2$ or after reaching DAS28 $<3.2$ after 12 weeks of therapy. The PREMIER Study, ${ }^{13}$ a multicenter randomized double-blind clinical trial of combination therapy with adalimumab plus MTX, enrolled 799 patients with early aggressive RA. ${ }^{12}$ Patients were randomized to one of three treatment groups: adalimumab plus MTX (20 mg/week), adalimumab plus placebo, and MTX plus placebo. After 2 years of treatment, the combination therapy adalimumab plus MTX resulted significantly superior to adalimumab alone or MTX alone in improving symptoms and signs of the disease, decreasing radiographic progression and, finally, favoring disease remission.

The percentages of patients reporting both serious adverse events (AEs) and infectious AEs were similar in the three groups of treatment. Nevertheless, the rate of serious infections in the adalimumab monotherapy group was significantly lower than that in the combination treatment group, but not different compared with the MTX monotherapy group. In particular, in the combination treatment arm, one female patient developed pleural tuberculosis (TB). This patient had no purified protein derivative test performed, had a negative chest X-ray at baseline, and did not receive primary prophylactic isoniazid before starting treatment. However, as we have previously reported, although TNF- $\alpha$ inhibitors are generally well-tolerated, physicians should be aware of the potential adverse events of these drugs. ${ }^{5-12}$ Indeed, TNF- $\alpha$ favors the recruitment and the activation of lymphocytes, neutrophils, and platelets, the expression of adhesion molecules (intercellular adhesion molecule-1 [ICAM-I], vascular cell adhesion molecule-1 [VCAM-1], selectins) on endothelial cells and induces the neo-angiogenesis in the sites of phlogosis. TNF has a central role in the initial host response to infection. ${ }^{6}$ In $\mathrm{TB}$, it results in macrophage activation, cell recruitment, granuloma formation, and maintenance of granuloma integrity. ${ }^{4,822}$ The overall risk of reactivation of 
latent TB and opportunistic infections should be considered before the beginning of therapy. Appropriate screening with the Mantoux test and chest X-ray should always be performed before starting treatment. Skin induration of $5 \mathrm{~mm}$ or greater should be interpreted as a positive result for latent TB (LTB) in any patient considered for anti-TNF- $\alpha$ treatment. However, the negative Mantoux test should be interpreted with caution in any patient who is under treatment with immunosuppressive drugs as they are more likely to have false-negative Mantoux test results. ${ }^{8}$

Accordingly, a number of TB cases have occurred in subjects who had a negative Mantoux test before starting TNF- $\alpha$ inhibitor therapy. ${ }^{8,23}$ By contrast, previous Bacillus Calmette-Guérin vaccination may cause false-positive Mantoux test results. However, the PREMIER Study confirmed that the patients at high risk for reactivation or primary TB infection must take isoniazid before starting treatment with adalimumab, as well as with the other TNF- $\alpha$ inhibitors. ${ }^{8,13}$ In the PREMIER Study, one case of lymphoma was reported in the MTX monotherapy arm, confirming the evidence that in RA patients, the risk for lymphoma is irrespective of treatment and does not exert a major influence over decisions to start or continue TNF- $\alpha$ inhibitor therapy in patients with rapidly progressing joint destruction. ${ }^{8,23}$ Furthermore, one case of lupus like reaction occurred in the combination treatment group. Increased frequencies of autoantibodies, such as antinuclear antibodies and anti-double-stranded DNA antibodies, have been reported in subjects under TNF- $\alpha$ inhibitor treatment. However, the risk for development of systemic autoimmune diseases is low, and at present there is no recommendation for the monitoring of autoantibody titers during anti-TNF- $\alpha$ therapy., ${ }^{8,24}$ Van der Heijde et a ${ }^{14}$ reported the data from 799 patients who participated in an ongoing open-label extension study of the PREMIER Study. Of these, 497 patients completed a further 3 years of open-label adalimumab therapy after the initial 2 years of double-blind treatment, allowing an analysis of a total of 5 years of treatment. The results obtained confirmed that the initial combination treatment with adalimumab plus MTX led to better long-term clinical, functional, and radiographic outcomes than either initial adalimumab alone or MTX alone. Only two cases of TB were reported, but no serious opportunistic infection AEs occurred. Only one case of lymphoma and one case of nonmelanoma skin cancer were reported.

Eleven cases of malignancies other than lymphoma and nonmelanoma skin cancer occurred. There were no serious AEs of lupus like syndrome or demyelinating disease reported. Therefore, the long-term efficacy and safety data suggest a favorable benefit-risk profile for the use of adalimumab for more than 5 years in patients with early RA. Pavelka et $\mathrm{al}^{25}$ presented and discussed the results of Phase 3 clinical trials using mAbs that inhibit T-lymphocyte activation, deplete B-lymphocytes or target cytokines such as TNF- $\alpha$ involved in the pathogenesis of RA in patients who did not respond to DMARDs. In particular, a pivotal 52-week, double-blind, placebo-controlled trial, 619 patients were randomized to receive placebo, adalimumab $20 \mathrm{mg}$ /week or adalimumab $40 \mathrm{mg}$ every other week plus their usual MTX dose. ${ }^{17}$ The study confirmed that the adding of adalimumab to the MTX regimen in patients partially responsive to MTX provided additional benefit with the inhibition of joint damage, reduction of symptoms and signs, and improvement in physical function. In the OPTIMA study, ${ }^{19} 1032$ patients with active RA were enrolled and assigned 1:1 to adalimumab plus MTX or placebo plus MTX for 26 weeks.

However, $44 \%$ of patients treated with adalimumab plus MTX and $24 \%$ of patients treated with placebo plus MTX achieved stable low disease activity, confirming that combination therapy was superior to MTX alone in obtaining higher American College of Rheumatology (ACR) criteria (ACR 20, 50, and 70) responses, more clinical remissions, greater mean decrease in DAS28, no radiographic progression, and normal functional status at week 26 . Furthermore, the frequencies of total AEs and serious AEs were similar between the two groups of therapy. In particular, one case of Pneumocystis jiroveci (carinii) pneumonia, two malignancies (malignant melanoma in situ and squamous cell carcinoma), and one case of lupus like syndrome occurred in the adalimumab plus MTX group.

Krieckaert et $\mathrm{al}^{26}$ enrolled 184 RA patients who were treated with adalimumab for at least 1 year. Bone mineral density (BMD) measurements of the total hip and lumbar spine were performed using dual-energy X-ray absorptiometry, and metacarpal cortex BMD was measured using digital X-ray radiogrammetry. This study demonstrated that the loss of BMD in the spine was arrested over 4 years of adalimumab therapy, whereas BMD of the hands and hip continued to decrease after 1 and 4 years, respectively. However, the changes in BMD were related to the disease activity and the current use of prednisone. Therefore, these results confirm and underline the need to monitor the degree of disease activity, not only for the long-term impact of the inflammation on local bone and the formation/progression of erosions, but also on the generalized bone loss, osteoporosis, and the additional risk of fractures. Finally, a few studies reported an increase in BMI during 2 years of therapy with TNF- $\alpha$ inhibitors. ${ }^{27,28}$ The increase in BMI may 
be favored by the release of adipocytokines, even if their actual role remains still not entirely clear. ${ }^{29}$ Takeuchi et al ${ }^{30}$ enrolled 334 Japanese patients with early RA (HOPEFUL 1 study) and randomized 171 patients to receive adalimumab plus MTX (6 mg-8 mg/week) and 163 to receive MTX plus placebo. The study confirmed that the combination adalimumab plus MTX regimen inhibited radiographic progression and favored the achievement of ACR20, ACR50, and ACR70. The study also confirmed the safety of adalimumab therapy. Indeed, there were no significant differences in the percentage of patients with AEs in the adalimumab plus MTX arm and in the MTXalone arm, and the incidence of serious AEs were rare. As is widely known, the synovium in RA is characterized by a dense infiltrate, consisting of T- and B-lymphocytes, plasma cells, macrophages, dendritic cells (DCs) and other cells. Chemerin is a recently discovered chemokine that specifically modulates chemotaxis and activation of macrophages and DCs (in particular plasmocytoid DCs and monocyte-derived DCs). ${ }^{31}$ Herenius et al ${ }^{32}$ measured the chemerin serum levels in 49 patients with active RA before and after 16 weeks of treatment with adalimumab. Adalimumab treatment decreased the chemerin serum levels which was also correlated with the decrease in DAS28, in serum levels of IL-6 and, finally, in the macrophage migration inhibitory factor. These findings confirm that adalimumab treatment promotes the improvement of the clinical parameters of disease activity. Notably, all studies confirmed the low incidence of cutaneous injection site reactions (ie, local erythema and swelling) as we have described. ${ }^{5-11}$ Finally, lymphotoxin (LT)- $\alpha$ seems to play a role in the development of flogosis of immune-mediated disease such as RA. Indeed, in human RA, in addition to TNF- $\alpha$, also lymphotoxin (LT)- $\alpha$ expression in the synovium is elevated. ${ }^{33}$ T-helper(Th)-1 and Th-17 lymphocytes have been associated with autoimmune diseases, such as RA, and expressed LT- $\alpha .{ }^{34,35}$ Depletion of LT- $\alpha$-expressing Th- 1 and Th-17 lymphocytes with LT- $\alpha$-specific mAb may be beneficial in the treatment of autoimmune disease such as RA. ${ }^{35}$ However, is it possible that adalimumab could bind LT- $\alpha$ as etanercept as demonstrated in PsA patients? ${ }^{36}$ Further studies are required to better define the role of LT- $\alpha$ and LT- $\alpha$ blockade both in PsA and RA patients.

\section{Potential beneficial effects on endothelial dysfunction and accelerated atherosclerosis in RA}

Autoimmune rheumatic diseases have been associated with accelerated atherosclerosis and various types of vasculopathies. ${ }^{34}$ The terms "endothelial activation" and "dysfunction" are used to describe the changes in endothelial homeostatic control mechanisms. Indeed, in response to a variety of noxious stimuli, endothelium undergoes a phenotypic modulation from the normal state to a nonadaptive state known as endothelial dysfunction. This pathophysiological condition is associated with increased expression of adhesion molecules such as Intercellullar adhesion molecule (ICAM)-1, vascular cellular adhesion molecule (VCAM)-1 and selectins, ${ }^{37-41}$ and pro-inflammatory cytokines (ie, TNF- $\alpha$, IL-1, IL-6, and interferon- $\gamma$ ) and prothrombotic factors as well as with oxidative stress upregulation and abnormal vascular tone modulation. Systemic chronic inflammation may add to excessive oxidative stress leading to the formation and accumulation of advanced glycation end products. It is known that several systems generating reactive oxygen species (ROS) may catalyze a variety of modifications to nucleic acids, lipids, and proteins favoring the appearance of neo-cryptic epitopes which may behave as autoantigens. ${ }^{34-37}$

Furthermore, vascular endothelial growth factor (VEGF) is a critical mediator of inflammation both in chronic immune-mediated and allergic diseases. ${ }^{37,41-43}$ It is known that VEGF is a pro-angiogenic factor which alters the microvascular network and, thus, correlates and may contribute to the development and progression of atherosclerosis. Indeed, it is now accepted that atherosclerosis is an inflammatory condition which starts as a "response to injury" that adds to traditional cardiovascular and genetic risk factors favoring endothelial dysfunction which arises before the appearance of the first morphological signs of atherosclerosis. The increase of common carotid intima-media thickness (ccIMT) and the impairment of brachial artery flow-mediated (FMD) and nitroglycerinemediated (NMD) vasodilatation represent, with the above reported methodological limitations, good early indicators of accelerated atherosclerosis in RA and SLE patients with negative history for vascular disease. ${ }^{37,44-47}$ However, Kerekes et $\mathrm{al}^{44}$ confirmed that TNF- $\alpha$ inhibitors (etanercept, infliximab, adalimumab) may have a beneficial effect on arterial stiffness in patients with RA slowing the progression of accelerated atherosclerosis and consequently reducing the cardiovascular risk. Szekanecz et $\mathrm{al}^{48}$ suggested that etanercept and adalimumab may exert beneficial effects on the lipid profile improving the endothelial dysfunction. Furthermore, TNF- $\alpha$ inhibitors are able to reduce the expression and production of VEGF, nitric oxide (NO), and inducible NO synthase. ${ }^{38}$ Gonzalez-Juanatey et al ${ }^{49}$ treated 34 RA patients with adalimumab and analyzed FMD values at baseline and after 12 months of therapy. 


\section{Adalimumab}

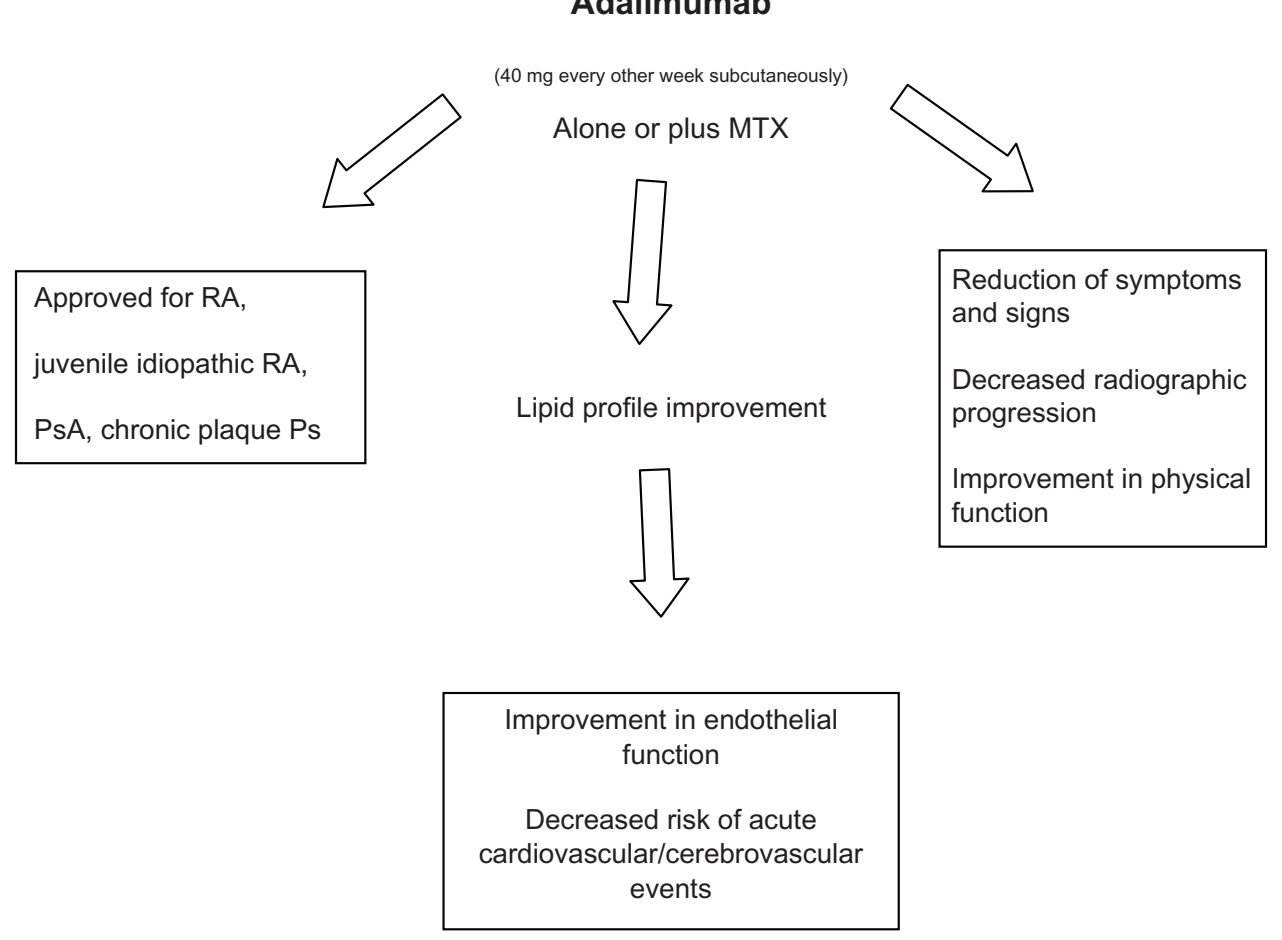

Figure I Therapeutic indications and beneficial effects of adalimumab.

Abbreviations: MTX, methotrexate; RA, rheumatoid arthritis; PsA, psoriatic arthritis; Ps, psoriasis.

Adalimumab treatment favored persistent improvement of endothelial function as proven by the increase in FMD value after 12 months of therapy.

In summary, the administration of TNF- $\alpha$ inhibitors reduces the systemic inflammation in patients with chronic immune-mediated diseases, improves both the clinical course of the disease itself and the endothelial function, and thus may decrease the risk of acute cardiovascular and/or cerebrovascular events.

Therapeutic indications and beneficial effects of adalimumab are depicted in Figure 1.

\section{Immunogenicity}

Although adalimumab sequence is fully humanized and it is supposed to be less immunogenic than murine or chimeric monoclonal antibodies, the production of antibodies to adalimumab has been reported, and it still remains unclear which part of its molecule induces the antibody response. ${ }^{8,50}$ However, the production of antibodies to adalimumab may reduce the efficacy of the drug and induce the development of adverse drug-reactions and exanthema. ${ }^{8,50}$

\section{Conclusion}

TNF- $\alpha$ inhibitors represent a new class of drugs which have revolutionized the clinical management of chronic inflammatory diseases such as RA. Moreover, adalimumab as the other TNF- $\alpha$ inhibitors may favor the improvement of the endothelial dysfunction, and, thus, reduce the risk of cardiovascular and cerebrovascular diseases. However, physicians need to be aware of the potential efficacy and risks of treatment with these agents.

\section{Disclosure}

The authors declare no conflicts of interest in this work.

\section{References}

1. Filipovic I, Walker D, Forster F, Curry AS. Quantifying the economic burden of productivity loss in rheumatoid arthritis. Rheumatology (Oxford). 2011;50(6):1083-1090.

2. Scott DL, Wolfe F, Huizinga TW. Rheumatoid arthritis. Lancet. 2010; 376(9746):1094-1108.

3. Takeuchi T. Revolutionary change in rheumatoid arthritis management with biological therapy. Keio J Med. 2011;60(3):75-81.

4. Peschon JJ, Torrance DS, Stocking KL, et al. TNF receptor-deficient mice reveal divergent roles for $\mathrm{p} 55$ and $\mathrm{p} 75$ in several models of inflammation. J Immunol. 1998;160(2):943-952.

5. Murdaca G, Colombo BM, Cagnati P, Gulli R, Spanò F, Puppo F. Update upon efficacy and safety of TNF- $\alpha$ inhibitors. Expert Opin Drug Saf. 2012;11(1):1-5.

6. Murdaca G, Colombo BM, Puppo F. Emerging biological drugs: a new therapeutic approach for systemic lupus erythematosus. An update upon efficacy and adverse events. Autoimmun Rev. 2011;11(1):56-60.

7. Murdaca G, Colombo BM, Puppo F. Adalimumab for the treatment of immune-mediated diseases: an update on old and recent indications. Drugs Today (Barc). 2011;47(4):277-288. 
8. Murdaca G, Colombo BM, Puppo F. Anti-TNF-alpha inhibitors: a new therapeutic approach for inflammatory immune-mediated diseases: an update upon efficacy and adverse events. Int $J$ Immunopathol Pharmacol. 2009;22(3):557-565.

9. Murdaca G, Colombo BM, Barabino G, Caiti M, Cagnati P, Puppo F. Anti-tumor necrosis factor- $\alpha$ treatment with infliximab for disseminated granuloma annulare. Am J Clin Dermatol. 2010;11(6):437-439.

10. Puppo F, Murdaca G, Ghio M, Indiveri F. Emerging biologic drugs for the treatment of rheumatoid arthritis. Autoimmun Rev. 2005;4(8): $537-541$.

11. Murdaca G, Spanò F, Puppo F. Selective TNF- $\alpha$ inhibitor-induced injection site reactions. Expert Opin Drug Saf. 2013;12(2):187-193.

12. Murdaca G, Spanò F, Miglino M, Puppo F. Effects of TNF- $\alpha$ inhibitors upon the mechanisms of action of VEGF. Immunotherapy. 2013;5(2): 113-115.

13. Breedveld FC, Weisman MH, Kavanaugh AF, et al. The PREMIER study: a multicenter, randomized, double-blind clinical trial of combination therapy with adalimumab plus methotrexate versus methotrexate alone or adalimumab alone in patients with early, aggressive rheumatoid arthritis who had not had previous methotrexate treatment. Arthritis Rheum. 2006;54(1):26-37.

14. van der Heijde D, Breedveld FC, Kavanaugh A, et al. Disease activity, physical function, and radiographic progression after long-term therapy with adalimumab plus methotrexate: 5-year results of PREMIER. J Rheumatol. 2010;37(11):2237-2246.

15. Weinblatt ME, Keystone EC, Furst DE, et al. Adalimumab, a fully human anti-tumor necrosis factor alpha monoclonal antibody, for the treatment of rheumatoid arthritis in patients taking concomitant methotrexate: the ARMADA trial. Arthritis Rheum. 2003;48(1):35-45.

16. van de Putte LB, Atkins C, Malaise M, et al. Efficacy and safety of adalimumab as monotherapy in patients with rheumatoid arthritis for whom previous disease modifying antirheumatic drug treatment has failed. Ann Rheum Dis. 2004;63(5):508-516.

17. Keystone EC, Kavanaugh AF, Sharp JT, et al. Radiographic, clinical, and functional outcomes of treatment with adalimumab (a human anti-tumor necrosis factor monoclonal antibody) in patients with active rheumatoid arthritis receiving concomitant methotrexate therapy: a randomized, placebo-controlled, 52-week trial. Arthritis Rheum. 2004;50(5):1400-1411.

18. Furst DE, Schiff MH, Fleischmann RM, et al. Adalimumab, a fully human anti-tumor necrosis factor-alpha monoclonal antibody, and concomitant standard antirheumatic therapy for the treatment of rheumatoid arthritis: results of STAR (Safety Trial of Adalimumab in Rheumatoid Arthritis). J Rheumatol. 2003;30(12):2563-2571.

19. Kavanaugh A, Fleischmann RM, Emery P, et al. Clinical, functional, and radiographic consequences of achieving stable low disease activity and remission with adalimumab plus methotrexate or methotrexate alone in early rheumatoid arthritis: 26-week results from the randomized, controlled OPTIMA study. Ann Rheum Dis. 2013;72(1): 64-71.

20. Chang J, Girgis L. Clinical use of anti-TNF-alpha biological agents a guide for GPs. Aust Fam Physician. 2007;36(12):1035-1038.

21. Inoue E, Yamanaka H, Hara M, Tomatsu T, Kamatani N. Comparison of Disease Activity Score (DAS)28-erythrocyte sedimentation rate and DAS28-C-reactive protein threshold values. Ann Rheum Dis. 2007;66(3):407-409.

22. Roach DR, Bean AG, Demangel C, France MP, Briscoe H, Britton WJ. TNF regulates chemokine induction essential for cell recruitment, granuloma formation, and clearance of mycobacterial infection. J Immunol. 2002;168(9):4620-4627.

23. Winthrop KL. Risk and prevention of tuberculosis and other serious opportunistic infections associated with the inhibition of tumor necrosis factor. Nat Clin Pract Rheumatol. 2006;2(11):602-610.

24. Gonnet-Gracia C, Barnetche T, Richez C, Blanco P, Dehais J, Schaeverbeke T. Antinuclear antibodies, anti-DNA, and C4 complement evolution in rheumatoid arthritis and ankylosing spondylitis treated with TNF-alpha blockers. Clin Exp Rheumatol. 2008;26(3):401-407.
25. Pavelka K, Kavanaugh AF, Rubbert-Roth A, Ferraccioli G. Optimizing outcomes in rheumatoid arthritis patients with inadequate responses to disease-modifying antirheumatic drugs. Rheumatology (Oxford). 2012;51 Suppl 5:v12-v21.

26. Krieckaert CL, Nurmohamed MT, Wolbink G, Lems WF. Changes in bone mineral density during long-term treatment with adalimumab in patients with rheumatoid arthritis: a cohort study. Rheumatology (Oxford). 2013;52(3):547-553.

27. Engvall IL, Tengstrand B, Brismar K, Hafström I. Infliximab therapy increases body fat mass in early rheumatoid arthritis independently of changes in disease activity and levels of leptin and adiponectin: a randomized study over 21 months. Arthritis Res Ther. 2010; 12(5):R197.

28. Briot K, Gossec L, Kolta S, Dougados M, Roux C. Prospective assessment of body weight, body composition, and bone density changes in patients with spondyloarthropathy receiving antitumor necrosis factor-alpha treatment. J Rheumatol. 2008;35(5):855-861.

29. Baker JF, George M, Baker DG, Toedter G, Von Feldt JM, Leonard MB. Associations between body mass, radiographic joint damage, adipokines and risk factors for bone loss in rheumatoid arthritis. Rheumatology (Oxford). 2011;50(11):2100-2107.

30. Takeuchi T, Yamanaka H, Ishiguro N, et al. Adalimumab, a human anti-TNF monoclonal antibody, outcome study for the prevention of joint damage in Japanese patients with early rheumatoid arthritis: the HOPEFUL 1 study. Ann Rheum Dis. Epub January 11, 2013.

31. Wittamer V, Grégoire F, Robberecht P, Vassart G, Communi D, Parmentier M. The C-terminal nonapeptide of mature chemerin activates the chemerin receptor with low nanomolar potency. J Biol Chem. 2004; 279(11):9956-9962.

32. Herenius MM, Oliveira AS, Wijbrandts CA, Gerlag DM, Tak PP, Lebre MC. Anti-TNF therapy reduces serum levels of chemerin in rheumatoid arthritis: a new mechanism by which anti-TNF might reduce inflammation. PLoS One. 2013;8(2):e57802.

33. Takemura S, Braun A, Crowson C, et al. Lymphoid neogenesis in rheumatoid synovitis. J Immunol. 2001;167(2):1072-1080.

34. Murdaca G, Colombo BM, Puppo F. The role of Th17 lymphocytes in the autoimmune and chronic inflammatory diseases. Intern Emerg Med. 2011;6(6):487-495.

35. Chiang EY, Kolumam GA, Yu X, et al. Targeted depletion of lymphotoxin-alpha-expressing TH1 and TH17 cells inhibits autoimmune disease. Nat Med. 2009;15(7):766-773.

36. Murdaca G, Colombo BM, Contini P, Puppo F. Determination of lymphotoxin-alpha levels in patients with psoriatic arthritis undergoing etanercept treatment. J Interferon Cytokine Res. 2012;32(6): 277-279.

37. Murdaca G, Colombo BM, Cagnati P, Gulli R, Spanò F, Puppo F. Endothelial dysfunction in rheumatic autoimmune diseases. Atherosclerosis. 2012;224(2):309-317.

38. Puddu P, Puddu GM, Cravero E, De Pascalis S, Muscari A. The emerging role of cardiovascular risk factor-induced mitochondrial dysfunction in atherogenesis. J Biomed Sci. 2009;16:112.

39. Buttari B, Profumo E, Mattei V, et al. Oxidized beta2-glycoprotein I induces human dendritic cell maturation and promotes a Thelper type 1 response. Blood. 2005;106(12):3880-3887.

40. Arvieux J, Regnault V, Hachulla E, Darnige L, Berthou F, Youinou P. Oxidation of beta2-glycoprotein I (beta2GPI) by the hydroxil radical alters phospholipid binding and modulates recognition by anti-beta2GPI autoantibodies. Thromb Haemost. 2001;86(4):1070-1076.

41. Murdaca G, Spanò F, Cagnati P, Puppo F. Free radicals and endothelial dysfunction: potential positive effects of TNF- $\alpha$ inhibitors. Redox report in press.

42. Ciprandi G, Murdaca G, Colombo BM, De Amici M, Marseglia GL. Serum vascular endothelial growth factor in allergic rhinitis and systemic lupus erythematosus. Hum Immunol. 2008;69(8):510-512.

43. Ciprandi G, Colombo BM, Murdaca G, De Amici M. Serum vascular endothelial growth factor and sublingual immunotherapy. Allergy. 2008;63(7):945-946. 
44. Kerekes G, Szekanecz Z, Dér H, et al. Endothelial dysfunction and atherosclerosis in rheumatoid arthritis: a multiparametric analysis using imaging techniques and laboratory markers of inflammation and autoimmunity. J Rheumatol. 2008;35(3):398-406.

45. Colombo BM, Cacciapaglia F, Puntoni M, et al. Traditional and nontraditional risk factors in accelerated atherosclerosis in systemic lupus erythematosus: role of vascular endothelial growth factor (VEGATS Study). Autoimmun Rev. 2009;8(4):309-315.

46. Colombo BM, Murdaca G, Caiti M, et al. Intima-media thickness: a marker of accelerated atherosclerosis in women with systemic lupus erythematosus. Ann N Y Acad Sci. 2007;1108:121-126.

47. Dulai R, Perry M, Twycross-Lewis R, Morrissey D, Atzeni F, Greenwald S. The effect of tumor necrosis factor- $\alpha$ antagonists on arterial stiffness in rheumatoid arthritis: a literature review. Semin Arthritis Rheum. 2012;42(1):1-8.
48. Szekanecz Z, Kerekes G, Soltész P. Vascular effects of biologic agents in RA and spondyloarthropathies. Nat Rev Rheumatol. 2009;5(12): 677-684.

49. Gonzalez-Juanatey C, Vazquez-Rodriguez TR, Miranda-Filloy JA, et al. Anti-TNF-alpha-adalimumab therapy is associated with persistent improvement of endothelial function without progression of carotid intima-media wall thickness in patients with rheumatoid arthritis refractory to conventional therapy. Mediators Inflamm. 2012;2012: 674265 .

50. Bender NK, Heilig CE, Dröll B, Wohlgemuth J, Armbruster FP, Heilig B. Immunogenicity, efficacy, and adverse events of adalimumab in RA patients. Rheumatol Int. 2007;27(3):269-274.

\section{Publish your work in this journal}

Open Access Rheumatology Research and Reviews is an international, peer-reviewed, open access journal, publishing all aspects of clinical and experimental rheumatology in the clinic and laboratory including the following topics: Pathology, pathophysiology of rheumatological diseases; Investigation, treatment and management of rheumatological diseases; Clinical trials and novel pharmacological approaches for the treatment of rheumatological disorders. The manuscript management system is completely online and includes a very quick and fair peerreview system, which is all easy to use. Visit http://www.dovepress.com/ testimonials.php to read real quotes from published authors.

Submit your manuscript here: http://www.dovepress.com/open-access-rheumatology-research-and-reviews-journal 